\title{
Complete Multipartite Graphs and Braess Edges
}

\author{
Yuxiang $\mathrm{Hu}$ and Steve Kirkland* \\ Department of Mathematics, University of Manitoba \\ Winnipeg, MB, R3T 2N2 \\ Canada
}

June 6, 2019

Keywords: Kemeny's constant; Random walk on a graph. 2010 AMS Subject Classification: 05C50, 15B51, 60J10.

\begin{abstract}
Given an irreducible stochastic matrix $T$, Kemeny's constant $\kappa(T)$ measures the expected number of steps from any initial state to a randomly chosen final state, and is thus regarded as an indicator of the overall transit efficiency of the corresponding Markov chain. For a random walk on a connected graph with twin vertices, we present a formula for the change in Kemeny's constant after the insertion of an edge between the twins. Using that result, we investigate the circumstances under which inserting a new edge increases Kemeny's constant. In particular, we characterise the complete multipartite graphs without dominating vertices having the property that adding any new edge increases Kemeny's constant. We establish an analogous result for complete split graphs. We also prove that if a complete multipartite graph has enough dominating vertices, then there is always a non-edge whose addition will decrease Kemeny's constant.
\end{abstract}

*Corresponding author; email: stephen.kirkland@umanitoba.ca . 


\section{Introduction and preliminaries}

New connections sometimes yield surprising results. A famous example of that effect is Braess' paradox [1, 2], whereby the addition of a new route in a vehicle traffic network can have the effect of increasing travel times. In this paper, we continue the study (initiated in [9]) of a version of Braess' paradox arising in Markov chains.

Next, we briefly introduce a few important results on Markov chains. Given a Markov chain, its transition matrix $T \in M_{n}(\mathbb{R})$ is a non-negative stochastic matrix with $t_{i, j}$ equal to the transition probability from state $i$ to state $j$ for $1 \leq i, j \leq n$. Thus, the chain can be completely studied by analysing the corresponding transition matrix. In particular, when $T$ is irreducible, the Perron-Frobenius theorem guarantees the existence of a unique probability vector $w^{t}$ such that $w^{t} T=T$. Known as the stationary distribution vector, $w^{t}$ captures long-term behaviour of the chain. Indeed, it is shown in [7] that

$$
\lim _{k \rightarrow \infty} \frac{1}{k} \sum_{j=0}^{k-1} \pi^{t} T^{j}=w^{t} .
$$

An important collection of quantities related to the short-term behaviour of a Markov chain is the set of mean first passage times: for each $1 \leq i, j \leq n$, the mean first passage time $m_{i, j}$ is the expected number of steps needed for a chain to arrive at state $j$ for the first time, given that it started in state $i$. Denoting the mean first passage time matrix by $M=\left[m_{i, j}\right]_{1 \leq i, j \leq n}$, a key observation by Kemeny [7] is that $M w=(\kappa(T)+1) \mathbb{1}$, where the constant $\kappa(T)$ is known as Kemeny's constant, and $\mathbb{1}$ denotes the all-ones column vector of appropriate order. The quantity $\kappa(T)+1=\sum_{j=1}^{n} m_{i, j} w_{j}$ $(1 \leq i \leq n)$ admits a natural interpretation - it is the expected number of steps, starting from state $i$, for the chain to arrive for the first time at a randomly chosen final state. Further, from the above, it is straightforward to see that $\kappa(T)+1=\sum_{i, j=1}^{n} w_{i} m_{i, j} w_{j}$, so that $\kappa(T)$ also measures the expected number of steps from a randomly chosen initial state to a randomly chosen final state. Thus, Kemeny's constant can be regarded as a measure of overall transit time for the Markov chain. As is shown in [7], Kemeny's constant can also be written as $\kappa(T)=\sum_{i=2}^{n} \frac{1}{1-\lambda_{i}}$ where $1, \lambda_{2}, \lambda_{3}, \ldots, \lambda_{n}$ are the eigenvalues for $T$. We note in passing that Kemeny's constant has arisen in a variety of applied settings, including consensus algorithms [6], economics [10], vehicle traffic networks [4] and wireless network design [5]. 
Of interest in the present paper is a special type of Markov chain, namely a random walk on a connected undirected graph $G$. Here the transition matrix can be written as $D^{-1} A$, where $A$ is the $(0,1)$ adjacency matrix of the graph, and $D$ is the diagonal matrix of vertex degrees. In this setting we will abuse the notation slightly and write $\kappa(G)$ instead of $\kappa\left(D^{-1} A\right)$. One might expect that adding a new edge into a graph $G$ would decrease the value of Kemeny's constant, but as shown in [9] that is not always the case. In this paper, we consider the notion of a Braess edge introduced in [9]: we say that a non-edge $e$ of $G$ is a Braess edge if its insertion strictly increases Kemeny's constant, i.e. if $\kappa(G \cup e)>\kappa(G)$. A non-edge $e$ is a non-Braess edge for $G$ if $\kappa(G \cup e) \leq \kappa(G)$. Indeed, one of the main results in [9] is that for almost every tree there is a Braess edge; in particular, every non-edge in a star graph of order at least 4 is a Braess edge.

Graphs with the property that every non-edge is a Braess edge may be instructive for the design of networks (such as wireless networks), for they not only illuminate the structure of graphs possessing Braess edges, but also generate further insight into the workings of Kemeny's constant. Consequently, we make the following definition: a connected graph $G$ is a Braess graph provided that every non-edge of $G$ is a Braess edge. Such graphs may be viewed as either annoying (since any edge addition makes Kemeny's constant worse), or appealing (since such graphs can be thought of providing local minima for Kemeny's constant). Motivated by the observation that $K_{1, n-1}$ is a Braess graph when $n \geq 4$, we seek larger families of Braess graphs, focusing in this paper on complete multipartite graphs.

In section 2 2 we identify two families of complete multipartite Braess graphs. In section 3.1, we provide a formula for the change in Kemeny's constant after the insertion of an edge between twin vertices. That result is used in sections 3.2 and 3.3 to discuss the complete multipartite graphs that are Braess graphs. In particular, Theorem 3.3.3 characterises the complete multipartite graphs without dominating vertices that are Braess graphs, while Conjecture 3.4 .7 speculates on the structure of complete multipartite graphs having dominating vertices that are Braess graphs.

Throughout we will use standard notation from linear algebra: $J_{k, j}$ and $0_{k, j}$ will denote the $k \times j$ all ones and all zeros matrices, respectively, $\mathbb{1}_{d}$ and $0_{d}$ will denote the all ones vector and the zero vector in $\mathbb{R}^{d}$, respectively, and $e_{j}$ will denote the $j$-th standard unit basis vector, whose order shall be clear from the context. 


\section{Complete bipartite graphs and complete split graphs}

Here we consider random walks on two families of graphs that admit Braess graphs: complete bipartite graphs, and complete split graphs. In this simplified setting, we will illustrate a technique that will be used throughout the paper and motivates our approach in section 3 to the general complete multipartite case.

Theorem 2.0.1. Suppose that $n \geq 4$ and consider the complete bipartite graph $G=K_{k, n-k}$, with $1 \leq k \leq n-1 . G$ is a Braess graph if and only if one of the following holds: (i) $k=1$, (ii) $k=n-1$, (iii) $\frac{3 n+8}{7}<k<\frac{4 n-8}{7}$ and $n=18,20,22$ or $n \geq 24$.

Proof. The cases $k=1$ and $k=n-1$ are both stars, and it follows from [9] that these are Braess graphs.

Next, we assume that $n-3 \geq k \geq 3$ (so that the sizes of the independent sets are at least 3 ) and let $T$ be the transition matrix given by

$$
T=\left[\begin{array}{c|c|c}
0_{2,2} & 0_{2, k-2} & \frac{1}{n-k} J_{2, n-k} \\
\hline 0_{k-2,2} & 0_{k-2, k-2} & \frac{1}{n-k} J_{k-2, n-k} \\
\hline \frac{1}{k} J_{n-k, 2} & \frac{1}{k} J_{n-k, k-2} & 0_{n-k, n-k}
\end{array}\right] .
$$

Notice that the partitioning of $T$ forms an equitable partition of the matrix [3], and the corresponding quotient matrix $Q$ is given by

$$
Q=\left[\begin{array}{ccc}
0 & 0 & 1 \\
0 & 0 & 1 \\
\frac{2}{k} & \frac{k-2}{k} & 0
\end{array}\right]
$$

When $k \geq 4$, the vectors

$t=\left[\begin{array}{c}1 \\ \frac{-1}{0_{k-2}} \\ 0_{n-k}\end{array}\right], u_{i}=\left[\frac{0_{2}}{\frac{e_{1}-e_{i}}{0_{n-k}}}\right], v_{j}=\left[\frac{0_{2}}{\frac{0_{k-2}}{e_{1}-e_{j}}}\right],(2 \leq i \leq k-2,2 \leq j \leq n-k)$

are null vectors of $T$, while when $k=3, T$ has null vectors $t, v_{2}, \ldots, v_{n-3}$; in either case we find that 0 is an eigenvalue of $T$ with multiplicity at least 
$n-3$. The remaining eigenvalues of $T$ are given by the eigenvalues of $Q$, i.e. $1,-1$, and 0 .

Now without loss of generality, suppose the edge inserted is between vertex 1 and 2. The resulting transition is given by

$$
\tilde{T}=\left[\begin{array}{cc|c|c}
0 & \frac{1}{n-k+1} & 0_{2, k-2} & \frac{1}{n-k+1} J_{2, n-k} \\
\frac{1}{n-k+1} & 0 & & \frac{1}{n-k} J_{k-2, n-k} \\
\hline 0_{k-2,2} & 0_{k-2, k-2} & \frac{1}{k} J_{n-k, k-2} & 0_{n-k, n-k}
\end{array}\right],
$$

with the corresponding quotient matrix

$$
\tilde{Q}=\left[\begin{array}{ccc}
\frac{1}{n-k+1} & 0 & \frac{n-k}{n-k+1} \\
0 & 0 & 1 \\
\frac{2}{k} & \frac{k-2}{k} & 0
\end{array}\right] .
$$

The vectors $t, u_{i}, 4 \leq i \leq k, v_{j}, k+2 \leq j \leq n$ are eigenvectors of $\tilde{T}$ corresponding to $-\frac{1}{n-k+1}$ and 0 , the latter with multiplicity $n-4$. The remaining eigenvalues of $\tilde{T}$ are those of the quotient matrix $\tilde{Q}$. Next we use the formula for Kemeny's constant in terms of eigenvalues in order to compute the change in Kemeny's constant when edge between vertices 1 and 2 is added to $K_{k, n-k}$. That is,

$$
\begin{aligned}
\kappa(\tilde{T})-\kappa(T) & =\kappa(\tilde{Q})-\kappa(Q)-\frac{1}{n-k+2} \\
& =\frac{7 k-3 n-8}{2 k n^{2}+\left(-4 k^{2}+4 k+2\right) n+2 k^{3}-4 k^{2}-2 k+4} \\
& =\frac{7 k-3 n-8}{2\left[k(n-k)^{2}+2 k(n-k)+n-k+2\right]} .
\end{aligned}
$$

Note that $\kappa(\tilde{T})-\kappa(T)>0$ if and only if $k>\frac{3 n+8}{7}$. Similarly, in order for any non-edge in the independent set of size $n-k$ to be Braess edge, we need $n-k>\frac{3 n+8}{7}$. We thus deduce that for $3 \leq k \leq n-3$ every non-edge is a Braess edge if and only if $\frac{3 n+8}{7}<k<\frac{4 n-8}{7}$. Note that the open interval $\left(\frac{3 n+8}{7}, \frac{4 n-8}{7}\right)$ includes a positive integer (which is necessarily at least 3 ) if and only if $n=18,20,22$ or $n \geq 24$. 
Lastly, when $k=2$, a computation reveals that $\kappa(\tilde{T})-\kappa(T)=\frac{n-1}{n}+$ $\frac{n-1}{2 n-3}-\frac{3}{2}<0$. The conclusion now follows.

Example 2.0.2. The graph $K_{8,8}$ serves as a 'boundary case' for Theorem 2.0.1. In the language of that theorem, we have $k=8$, and $n=2 k=16$. Observe that for these values of $k$ and $n$ we have $7 k=3 n+8=7(n-k)$. It now follows that adding an edge to $K_{8,8}$ will have no effect on Kemeny's constant for the corresponding random walk.

Recall that a complete split graph is formed by removing a clique on $j$ vertices from a complete graph on $n$ vertices; equivalently, a complete split graph can be written as $\bar{K}_{j} \vee K_{n-j}$, where $\vee$ denotes the join operation. We have the following result for complete split graphs; its proof follows along the same lines as that of Theorem 2.0.1, and is omitted.

Theorem 2.0.3. Suppose that $n \geq 4$ and the complete split graph $G=$ $\bar{K}_{j} \vee K_{n-j}$ where $2 \leq j \leq n-1 . \quad G$ is a Braess graph if and only if $\frac{14-6 n+\sqrt{68 n^{2}-8 n+4}}{8}<j \leq n-1$.

Example 2.0.4. It is straightforward to see that Theorem 2.0.3 is equivalent to the statement that $\bar{K}_{j} \vee K_{n-j}$ is a Braess graph if and only if $n-1 \geq j$ and $2 j^{2}+(3 n-7) j-\left(n^{2}+5 n-6\right)>0$. Further, for values of $j$ and $n$ such that $2 j^{2}+(3 n-7) j-\left(n^{2}+5 n-6\right)=0$, then adding an edge into $\bar{K}_{j} \vee K_{n-j}$ will have no effect on Kemeny's constant for the corresponding random walk. For instance $j=1170, n=4161$ is such a pair, and one may generate other such pairs by solving the corresponding Diophantine equation.

\section{Random walks on complete multipartite graphs}

\subsection{Graphs with twin vertices}

The results in section 2 conform to the pattern that a large independent set leads to the existence of Braess edges. Motivated by this intuition, it is natural to ask: are there any Braess graphs in the class of complete multipartite graphs $K_{k_{1}, \ldots, k_{r}}$ with $r \geq 3$ ? To address that question, we first develop some theory in a broader setting, namely, graphs with twin vertices.

Recall that in a graph $G$, two nonadjacent vertices $u$ and $v$ are called twins provided that their neighbourhoods coincide, i.e. a vertex $x$ is adjacent to $u$ in $G$ if and only if it is adjacent to $v$. In this section, in the setting of a 
random walk on a graph, we investigate the change in Kemeny's constant in the scenario where an extra edge is inserted between a pair of twin vertices.

First we provide an expression for the change of Kemeny's constant after perturbing one row of the transition matrix. This expression is fundamental to our subsequent results. As part of that expression, we make use of the group inverse $(I-T)^{\#}$ associated with an irreducible stochastic matrix $T$; we refer the reader to [8] for background on this group inverse and its use in the study of Markov chains. In particular we recall the following basic property: if $T$ has stationary vector $w^{t}$, then $(I-T)^{\#}(I-T)=I-\mathbb{1} w^{t}=(I-T)(I-T)^{\#}$.

Here we let $e_{i}$ denote the $i$-th standard unit basis vector in $\mathbb{R}^{n}$.

Lemma 3.1.1. Let $T$ be an irreducible, stochastic matrix of order $n$ and $\tilde{T}=T+e_{i} u^{t}$ where $u^{t}$ is some appropriate perturbing vector so that $\tilde{T}$ is still irreducible and stochastic. Let

$$
\theta_{j}=\frac{u^{t}(I-T)^{\#} e_{j}}{1-u^{t}(I-T)^{\#} e_{i}}, j=1, \ldots, n .
$$

Denote the stationary vector of $T$ by $w^{t}$, and the mean first passage times by $m_{p, q}(1 \leq p, q \leq n)$. Fix $k \neq i$. The change in Kemeny's constant is given by

$$
\kappa(\tilde{T})-\kappa(T)=w_{i} \sum_{j} \theta_{j}\left(m_{k, i}-m_{j, i}\right)+\theta_{i} .
$$

Proof. Denote the mean first passage times of $\tilde{T}$ by $\tilde{m}_{p, q}(1 \leq p, q \leq n)$, and the stationary vector of $\tilde{T}$ by $\tilde{w}^{t}$. From [8] Proposition $6.1 .7, \tilde{m}_{k, j}=$ $\frac{w_{j}}{w_{j}+\theta_{j} w_{i}} m_{k, j}+\frac{\theta_{j} w_{i}}{w_{j}+\theta_{j} w_{i}}\left(m_{k, i}-m_{j, i}\right)$, and $\tilde{m}_{k, i}=m_{k, i}$. Also, notice that

$$
\left(I-e_{i} u^{t}(I-T)^{\#}\right)^{-1}=I+\frac{1}{1-u^{t}(I-T)^{\#} e_{i}} e_{i} u^{t}(I-T)^{\#},
$$

so that

$$
\left.\tilde{w}^{t}=w^{t}\left(I-e_{i} u^{t}(I-T)^{\#}\right)^{-1}=w^{t}\left(I+\frac{1}{1-u^{t}(I-T)^{\#} e_{i}} e_{i} u^{t}(I-T)^{\#}\right)\right)
$$


from [8] Lemma 5.3.1. Therefore,

$$
\begin{aligned}
& \kappa(\tilde{T})-\kappa(T)=\sum_{j=1}^{n}\left(\tilde{m}_{k, j} \tilde{w}_{j}-m_{k, j} w_{j}\right) \\
& =\sum_{j \neq i, k}\left(\tilde{m}_{k, j} \tilde{w}_{j}-m_{k, j} w_{j}\right)+\left(\left(\tilde{m}_{k, i} \tilde{w}_{i}-m_{k, i} w_{i}\right)\right) \\
& =\sum_{j \neq i, k}\left[\left(\frac{w_{j}}{w_{j}+\theta_{j} w_{i}} m_{k, j}+\frac{\theta_{j} w_{i}}{w_{j}+\theta_{j} w_{i}}\left(m_{k, i}-m_{j, i}\right)\right) \times\right. \\
& \left.\left(w^{t}\left(I+\frac{1}{1-u^{t}(I-T)^{\#} e_{i}} e_{i} u^{t}(I-T)^{\#}\right)\right) e_{j}-m_{k, j} w_{j}\right] \\
& \left.+m_{k, i} w^{t}\left(I+\frac{1}{1-u^{t}(I-T)^{\#} e_{i}} e_{i} u^{t}(I-T)^{\#}\right)\right) e_{i}-m_{k, i} w_{i} \\
& =\sum_{j=1}^{n} \theta_{j} w_{i}\left(m_{k, i}-m_{j, i}\right)+\theta_{i},
\end{aligned}
$$

as desired.

To make use of Lemma 3.1.1, we use the same technique as shown in the bipartite case, namely an equitable partition, to refer our problem to the study of a transition matrix with one row perturbed.

For convenience, throughout this section, we denote the transition matrix of a random walk on an undirected graph of order $n$ with twins 1 and 2 having $d$ neighbours by

$$
T=\left[\begin{array}{c|c|c}
0_{2,2} & \frac{1}{d} J_{2, d} & 0_{2, n-d-2} \\
\hline x \mathbb{1}_{2}^{t} & A & B \\
\hline 0_{n-d-2,2} & C & D
\end{array}\right],
$$

(here $x$ is some positive vector in $\mathbb{R}^{d}$ ), and the transition matrix after the insertion of an edge between the twins by $\tilde{T}$. The quotient matrix of $T$ corresponding to the partition $\{\{1,2\},\{3\}, \ldots,\{n\}\}$ is given by the block matrix

$$
Q=\left[\begin{array}{c|c|c}
0 & \frac{1}{d} \rrbracket_{d}^{t} & 0_{n-d-2}^{t} \\
\hline 2 x & A & B \\
\hline 0_{n-d-2} & C & D
\end{array}\right] .
$$


Recall that the eigenvalues of $T$ consist of the eigenvalues of $Q$, along with 0 . Denote the stationary vector of $Q$ by $w^{t}$, and the mean first passage times of $Q$ by $M=\left[m_{i, j}\right]_{1 \leq i, j \leq n-1}$.

Theorem 3.1.2. Suppose that $T$ is given by (1), and that $\tilde{T}$ is the transition matrix arising from the addition of the edge between the twin vertices 1,2. Then the change in Kemeny's constant is given by

$$
\kappa(\tilde{T})-\kappa(T)=\frac{w_{1}}{d+w_{1}}\left(\sum_{j=1}^{n} w_{j} m_{j, 1}-1-m_{1,1}+\frac{2-w_{1}}{w_{1}(d+2)}\right) .
$$

Proof. The corresponding quotient matrix after the insertion of the extra edge is given by

$$
\tilde{Q}=\left[\begin{array}{c|c|c}
\frac{1}{d+1} & \frac{1}{d+1} \rrbracket_{d}^{t} & 0_{n-d-2}^{t} \\
\hline 2 x & A & B \\
\hline 0_{n-d-2} & C & D
\end{array}\right]
$$

and the eigenvalues of $\tilde{T}$ consist of the eigenvalues of $\tilde{Q}$, along with $-\frac{1}{d+1}$. Note that $\tilde{Q}=Q+e_{1} u^{t}$ where

$$
u^{t}=\frac{1}{d+1}\left[1\left|-\frac{1}{d} \rrbracket_{d}^{t}\right| 0_{n-d-2}^{t}\right]=\frac{1}{d+1} e_{1}^{t}(I-Q) .
$$

Notice that

$u^{t}(I-Q)^{\#}=\frac{1}{d+1} e_{1}^{t}(I-Q)(I-Q)^{\#}=\frac{1}{d+1} e_{1}^{t}\left(I-\mathbb{1} w^{t}\right)=\frac{1}{d+1}\left(e_{1}^{t}-w^{t}\right)$.

Let $\theta_{j}=\frac{u^{t}(I-Q)^{\#} e_{j}}{1-u^{t}(I-Q)^{\#} e_{1}}=\frac{e_{1}^{t} e_{j}-w_{j}}{d+w_{1}}$. Similar to Theorem 2.0.1 and applying Lemma 3.1.1, we have (taking $k=2$ in that lemma)

$$
\begin{aligned}
& \kappa(\tilde{T})-\kappa(T)=\kappa(\tilde{Q})-\kappa(Q)+\frac{1}{1-\left(-\frac{1}{d+1}\right)}-\frac{1}{1-0} \\
& =\kappa(\tilde{Q})-\kappa(Q)-\frac{1}{d+2} \\
& =w_{1} \sum_{j=1}^{n} \theta_{j}\left(m_{2,1}-m_{j, 1}\right)+\theta_{1}-\frac{1}{d+2} \\
& =w_{1}\left(\sum_{j=1}^{n} \frac{e_{1}^{t} e_{j}-w_{j}}{d+w_{1}}\left(m_{2,1}-m_{j, 1}\right)\right)+\theta_{1}-\frac{1}{d+2} \\
& =\frac{w_{1}}{d+w_{1}}\left(\sum_{j=1}^{n}\left(w_{j} m_{j, 1}-1-m_{1,1}+\frac{2-w_{1}}{w_{1}(d+2)}\right)\right) .
\end{aligned}
$$


Next we utilise Theorem 3.1.2 in our investigation of Braess edges for complete multipartite graphs.

\subsection{Theorem 3.1.2 for complete multipartite graphs}

Let $T$ be the transition matrix of the random walk on a complete multipartite undirected graph with independent sets $S_{1}, S_{2}, \ldots, S_{r}$ of sizes $k_{1}, k_{2}, \ldots, k_{r}$ respectively, and assume $k_{1} \geq 2$. Let $\tilde{T}$ be the transition matrix after inserting an edge in $S_{1}$, say the edge $1 \sim 2$. Let $Q$ be the quotient matrix of $T$ induced by vertex sets $\{1,2\}, S_{1} \backslash\{1,2\}$ (if this is non-empty), $S_{2}, S_{3}, \ldots, S_{r}$. To study the change in Kemeny's constant, we derive an expression in terms of the sizes of independent sets.

Here we introduce some notation that is useful throughout this section. Let $n=\sum_{j=1}^{r} k_{j}, \alpha_{j}=n-k_{j}(1 \leq j \leq r), \gamma=\sum_{j=1}^{r} k_{j} \alpha_{j}$. Since the value of $k_{1}$ determines the way the graph is partitioned (when $k_{1}=2$, the subset $S_{1} \backslash\{1,2\}$ vanishes), we first deal with the case when $k_{1} \geq 3$. The case that $k_{1}=2$ will follow similarly.

Case 1: $k_{1} \geq 3$

Without loss of generality, assume that

$T=\left[\begin{array}{c|c|c|c|c|c}0_{2,2} & 0_{2, k_{1}-2} & \frac{1}{\alpha_{1}} J_{2, k_{2}} & \frac{1}{\alpha_{1}} J_{2, k_{3}} & \ldots & \frac{1}{\alpha_{1}} J_{2, k_{r}} \\ \hline 0_{k_{1}-2,2} & 0_{k_{1}-2, k_{1}-2} & \frac{1}{\alpha_{1}} J_{k_{1}-2, k_{2}} & \frac{1}{\alpha_{1}} J_{k_{1}-2, k_{3}} & \ldots & \frac{1}{\alpha_{1}} J_{k_{1}-2, k_{r}} \\ \hline \frac{1}{\alpha_{2}} J_{k_{2}, 2} & \frac{1}{\alpha_{2}} J_{k_{2}, k_{1}-2} & 0_{k_{2}, k_{2}} & \frac{1}{\alpha_{2}} J_{k_{2}, k_{3}} & \ldots & \frac{1}{\alpha_{2}} J_{k_{2}, k_{r}} \\ \hline \vdots & \vdots & \vdots & \ddots & \vdots & \vdots \\ \hline \frac{1}{\alpha_{r-1}} J_{k_{r-1}, 2} & & \ldots & & 0_{k_{r-1}, k_{r-1}} & \frac{1}{\alpha_{r-1}} J_{k_{r-1}, k_{r}} \\ \hline \frac{1}{\alpha_{r}} J_{k_{r}, 2} & \ldots & \ldots & \ldots & \ldots & 0_{k_{r}, k_{r}}\end{array}\right]$,

so that the corresponding quotient matrix is

$$
Q=\left[\begin{array}{cccccc}
0 & 0 & \frac{k_{2}}{\alpha_{1}} & \frac{k_{3}}{\alpha_{1}} & \ldots & \frac{k_{r}}{\alpha_{1}} \\
0 & 0 & \frac{k_{2}}{\alpha_{1}} & \frac{k_{3}}{\alpha_{1}} & \ldots & \frac{k_{r}}{\alpha_{1}} \\
\frac{2}{\alpha_{2}} & \frac{k_{1}-2}{\alpha_{2}} & 0 & \frac{k_{3}}{\alpha_{2}} & \ldots & \frac{k_{r}}{\alpha_{2}} \\
\vdots & \vdots & \vdots & \ddots & \ldots & \vdots \\
\frac{2}{\alpha_{r}} & \frac{k_{1}-2}{\alpha_{r}} & \frac{k_{2}}{\alpha_{r}} & \frac{k_{3}}{\alpha_{r}} & \ldots & 0
\end{array}\right]
$$

To apply Theorem 3.1.2, we need to find the stationary distribution and the mean first passage times into state 1 for $Q$. 
Proposition 3.2.1. Suppose that $Q$ is given by (3). Then the stationary vector of $Q$ is given by

$$
w^{t}=\frac{1}{\gamma}\left[\begin{array}{llll}
2 \alpha_{1} & \left(k_{1}-2\right) \alpha_{1} & k_{2} \alpha_{2} & k_{3} \alpha_{3} \ldots k_{r} \alpha_{r}
\end{array}\right] .
$$

Proof. A direct computation reveals that $w^{t} Q=w^{t}$.

Proposition 3.2.2. Suppose that $Q$ is given by (3). The mean first passage times into vertex 1 (corresponding to $\{1,2\}$ in the original graph) of the quotient matrix $Q$ are given by

$$
\left[\begin{array}{c}
m_{1,1} \\
m_{2,1} \\
m_{3,1} \\
\vdots \\
m_{r+1,1}
\end{array}\right]=\left[\begin{array}{c}
\frac{\gamma}{2 \alpha_{1}} \\
\frac{\gamma}{2 \alpha_{1}} \\
\frac{\alpha_{2}}{n}+\frac{k_{1}-2}{2}+\frac{(n-2)\left(\gamma-k_{1} \alpha_{1}\right)}{2 n \alpha_{1}} \\
\vdots \\
\frac{\alpha_{r}}{n}+\frac{k_{1}-2}{2}+\frac{(n-2)\left(\gamma-k_{1} \alpha_{1}\right)}{2 n \alpha_{1}}
\end{array}\right]
$$

Proof. From [7] Theorem 3.2.4,

$$
\left[\begin{array}{c}
m_{2,1} \\
m_{3,1} \\
\vdots \\
m_{r+1,1}
\end{array}\right]=\left(I-Q_{(1)}\right)^{-1} \mathbb{1}_{r-1}
$$

where $Q_{(1)}$ is the principal submatrix of $Q$ formed by deleting the first row and column. Notice that $\left(I-Q_{(1)}\right)=A-u v^{t}$ where

$$
A=\left[\begin{array}{ccccc}
1+\frac{k_{1}-2}{\alpha_{1}} & & & & 0 \\
& 1+\frac{k_{2}}{\alpha_{2}} & & & \\
& & 1+\frac{k_{3}}{\alpha_{3}} & & \\
0 & & & \ddots & 1+\frac{k_{r}}{\alpha_{r}}
\end{array}\right]
$$

is a diagonal matrix, and

$$
u=\left[\begin{array}{c}
\frac{1}{\alpha_{1}} \\
\frac{1}{\alpha_{2}} \\
\vdots \\
\frac{1}{\alpha_{r}}
\end{array}\right], v^{t}=\left[\begin{array}{lllll}
k_{1}-2 & k_{2} & k_{3} & \ldots & k_{r}
\end{array}\right] .
$$


Applying the Sherman-Morrison formula, we obtain

$$
\left(I-Q_{(1)}\right)^{-1}=\left(A-u v^{t}\right)^{-1}=A^{-1}+\frac{A^{-1} u v^{t} A^{-1}}{1-v^{t} A^{-1} u}
$$

and the conclusion follows immediately.

Theorem 3.2.3. Suppose that $T$ is given by (2) and that $\tilde{T}$ is the transition matrix arising from adding the edge between vertices 1 and 2 . Then $\kappa(\tilde{T})-$ $\kappa(T)=\frac{2}{\gamma+2}\left\{-\frac{\gamma}{2 \alpha_{1}}+\frac{1}{\gamma}\left[\frac{\left(k_{1}-2\right) \gamma}{2}+\sum_{j=2}^{r} k_{j} \alpha_{j}\left(\frac{\alpha_{j}}{n}+\frac{k_{1}-2}{2}+\frac{(n-2)\left(\gamma-k_{1} \alpha_{1}\right)}{2 n \alpha_{1}}\right)\right]+\frac{\gamma-\alpha_{1}}{\alpha_{1}\left(\alpha_{1}+2\right)}\right\}$.

Proof. Applying Theorem 3.1.2, Proposition 3.2.1, and Proposition 3.2.2, the result follows immediately.

Case 2: $k_{1}=2$

Without loss of generality, assume that

$$
T=\left[\begin{array}{c|c|c|cc}
0_{2,2} & \frac{1}{\alpha_{1}} J_{2, k_{2}} & \frac{1}{\alpha_{1}} J_{2, k_{3}} & \ldots & \frac{1}{\alpha_{1}} J_{2, k_{r}} \\
\hline \frac{1}{\alpha_{2}} J_{k_{2}, 2} & 0_{k_{2}, k_{2}} & \frac{1}{\alpha_{2}} J_{k_{2}, k_{3}} & \ldots & \frac{1}{\alpha_{2}} J_{k_{2}, k_{r}} \\
\vdots & \vdots & \ddots & \vdots & \vdots \\
\hline \frac{1}{\alpha_{r}} J_{k_{r}, 2} & \cdots & \cdots & \cdots & 0_{k_{r}, k_{r}}
\end{array}\right]
$$

so that the corresponding quotient matrix is

$$
Q=\left[\begin{array}{ccccc}
0 & \frac{k_{2}}{\alpha_{1}} & \frac{k_{3}}{\alpha_{1}} & \ldots & \frac{k_{r}}{\alpha_{1}} \\
\frac{2}{\alpha_{2}} & 0 & \frac{k_{3}}{\alpha_{2}} & \ldots & \frac{k_{r}}{\alpha_{2}} \\
\vdots & \vdots & \ddots & \ldots & \vdots \\
\frac{2}{\alpha_{r}} & \frac{k_{2}}{\alpha_{r}} & \frac{k_{3}}{\alpha_{r}} & \ldots & 0
\end{array}\right]
$$

The proof of the following is analogous to the proofs of Propositions 3.2 .1 and 3.2.2, and is omitted.

Proposition 3.2.4. Suppose that $Q$ is given by (5). Then the stationary vector for $Q$ is given by

$$
w^{t}=\frac{1}{\gamma}\left[\begin{array}{lll}
2 \alpha_{1} & k_{2} \alpha_{2} & k_{3} \alpha_{3} \ldots k_{r} \alpha_{r}
\end{array}\right]
$$


The mean first passage times into vertex 1 (corresponding to $\{1,2\}$ in the original graph) of the quotient matrix $Q$ is given by

$$
\left[\begin{array}{c}
m_{1,1} \\
m_{2,1} \\
m_{3,1} \\
\vdots \\
m_{r, 1}
\end{array}\right]=\left[\begin{array}{c}
\frac{\gamma}{2 \alpha_{1}} \\
\left(\alpha_{2}+\frac{\gamma-2 \alpha_{1}}{2}\right) \frac{1}{n} \\
\left(\alpha_{3}+\frac{\gamma-2 \alpha_{1}}{2}\right) \frac{1}{n} \\
\vdots \\
\left(\alpha_{r}+\frac{\gamma-2 \alpha_{1}}{2}\right) \frac{1}{n}
\end{array}\right]
$$

Theorem 3.2.5. Suppose that $T$ is given by (4) and that $\tilde{T}$ is the transition matrix arising from adding the edge between vertices 1,2 . Then

$$
\kappa(\tilde{T})-\kappa(T)=\frac{2}{\gamma+2}\left(\frac{1}{\gamma n} \sum_{j=2}^{r} k_{j} \alpha_{j}^{2}+\frac{\left(\gamma-2 \alpha_{1}\right)^{2}}{2 \gamma n}-\frac{\gamma}{2 \alpha_{1}}+\frac{\gamma-\alpha_{1}}{\left(\alpha_{1}+2\right) \alpha_{1}}\right) .
$$

Proof. Applying Theorem 3.1.2 and Proposition 3.2.4 the result follows immediately.

\subsection{Existence of non-Braess edges in complete multi- partite graphs}

As mentioned previously, it follows from [9] that every non-edge in a star graph of order at least 4 is a Braess edge. The results of section 2 characterise the complete split graphs and complete bipartite graphs for which every nonedge is a Braess edge, thus generalising the observation for star graphs. In this section we consider general complete multipartite graphs, as another natural generalisation of star graphs.

Theorem 3.3.1. Suppose that $G=K_{k_{1}, k_{2}, \ldots, k_{r}}$ is a complete $r$-partite with $r \geq 3$. Suppose further that $\min \left\{k_{1}, \ldots, k_{r}\right\} \geq 3$. Then $G$ has a non-Braess edge.

Proof. Without loss of generality, suppose that $k_{1}$ is the smallest among all the $k_{i}$ 's $(1 \leq i \leq r)$, so that $\alpha_{1}$ is the greatest among all the $\alpha_{j}$ 's $(1 \leq j \leq r)$. 
From Theorem 3.2 .3 ,

$$
\begin{aligned}
& \kappa(\tilde{T})-\kappa(T) \leq \frac{2}{\gamma+2}\left\{-\frac{\gamma}{2 \alpha_{1}}+\frac{1}{\gamma}\left[\frac{\left(k_{1}-2\right) \gamma}{2}\right.\right. \\
& \left.\left.+\sum_{j=2}^{r} k_{j} \alpha_{j}\left(\frac{\alpha_{1}}{n}+\frac{k_{1}-2}{2}+\frac{(n-2)\left(\gamma-k_{1} \alpha_{1}\right)}{2 n \alpha_{1}}\right)\right]+\frac{\gamma-\alpha_{1}}{\alpha_{1}\left(\alpha_{1}+2\right)}\right\} \\
& =\frac{2}{\gamma+2}\left(-\frac{\gamma}{2 \alpha_{1}}+\frac{k_{1}-2}{2}+\frac{(n-2)\left(\gamma-k_{1} \alpha_{1}\right)}{2 \alpha_{1} n}+\frac{\gamma-\alpha_{1}}{\alpha_{1}\left(\alpha_{1}+2\right)}\right) \\
& =\frac{2}{\gamma+2}\left(\frac{-6 \alpha_{1} n-2 \alpha_{1}^{2} n-2 \alpha_{1} \gamma+2 k_{1} \alpha_{1}^{2}-4 \gamma+4 k_{1} \alpha_{1}+2 n \gamma}{2 \alpha_{1}\left(\alpha_{1}+2\right) n}\right) \\
& =\frac{2}{\gamma+2}\left(\frac{4\left(k_{1} \alpha_{1}-\gamma\right)+2\left(-\alpha_{1}^{3}+\gamma k_{1}\right)-6 \alpha_{1} n}{2 \alpha_{1}\left(\alpha_{1}+2\right) n}\right) .
\end{aligned}
$$

To show that this last expression is negative, it suffices to observe that

$$
\begin{aligned}
-\alpha_{1}^{3}+\gamma k_{1} & =-\left(\sum_{j=2}^{r} k_{j}^{2}+2 \sum_{2 \leq i \neq j \leq r} k_{i} k_{j}\right) \alpha_{1}+2 k_{1}\left(\sum_{2 \leq i \neq j \leq r} k_{i} k_{j}+k_{1} \alpha_{1}\right) \\
& =2\left(k_{1}-\alpha_{1}\right) \sum_{2 \leq i \neq j \leq r} k_{i} k_{j}+\left(2 k_{1}^{2} \alpha_{1}-\sum_{j=2}^{r} k_{j}^{2} \alpha_{1}\right) \leq 0 .
\end{aligned}
$$

Theorem 3.3.2. Suppose that $G=K_{k_{1}, k_{2}, \ldots, k_{r}}$ is a complete $r$-partite with $r \geq 3$. Suppose further that $\min \left\{k_{1}, \ldots, k_{r}\right\}=2$. Then $G$ has a non-Braess edge.

Proof. Without loss of generality, suppose $k_{1}=2$, so that $\alpha_{1}$ is the greatest among all the $\alpha_{j}$ 's $(1 \leq j \leq r)$. From Theorem 3.2 .5 we have,

$$
\begin{aligned}
\kappa(\tilde{T})-\kappa(T) & =\frac{2}{\gamma+2}\left(\frac{1}{\gamma n} \sum_{j=2}^{r} k_{j} \alpha_{j}^{2}+\frac{\left(\gamma-2 \alpha_{1}\right)^{2}}{2 \gamma n}-\frac{\gamma}{2 \alpha_{1}}+\frac{\gamma-\alpha_{1}}{\left(\alpha_{1}+2\right) \alpha_{1}}\right) \\
& \leq \frac{2}{\gamma+2}\left(\frac{\alpha_{1}}{\gamma n}\left(\gamma-2 \alpha_{1}\right)+\frac{\left(\gamma-2 \alpha_{1}\right)^{2}}{2 \gamma n}-\frac{\gamma}{2 \alpha_{1}}+\frac{\gamma-\alpha_{1}}{\left(\alpha_{1}+2\right) \alpha_{1}}\right) \\
& =\frac{2}{\gamma+2}\left(\frac{\gamma-2 \alpha_{1}}{2 n}+\frac{-\gamma-2}{2\left(\alpha_{1}+2\right)}\right) \\
& =\frac{2}{\gamma+2}\left(\frac{-2 \alpha_{1}^{2}-4 \alpha_{1}-2 n}{2 n\left(\alpha_{1}+2\right)}\right)<0 .
\end{aligned}
$$


The following theorem summarises some of our results so far. Its proof is immediate from Theorems 2.0.1, 3.3.1 and 3.3.2.

Theorem 3.3.3. Suppose that $G=K_{k_{1}, \ldots, k_{r}}$ is a complete multipartite graph such that $r \geq 2$ and $\min \left\{k_{1}, \ldots, k_{r}\right\} \geq 2$. Then $G$ is a Braess graph if and only if $r=2, k_{1}+k_{2}=18,20,22$ or $k_{1}+k_{2} \geq 24$, and $\frac{3 k_{2}+8}{4}<k_{1}<\frac{4 k_{2}-8}{3}$.

\subsection{Complete multipartite graphs with dominating ver- tices}

Recall that a dominating vertex in a graph is one that is adjacent to every other vertex in the graph. While Theorem 2.0.3 identifies a certain family of complete multipartite graphs with dominating vertices that are Braess graphs, the following example highlights the fact that there are some subtleties inherent in characterising such graphs in general.

Example 3.4.1. For the complete multipartite graph $G=K_{9,9,1,1}$, we have $\kappa(G)=\frac{4339}{236}$. If we add any edge to $G$ to create $\tilde{G}$, we find that $\kappa(\tilde{G})=$ $\frac{1287}{70}>\kappa(G)$. Consequently $K_{9,9,1,1}$ is a Braess graph.

Next we consider $H=K_{9,9,1,1,1}$, and find that $\kappa(H)=\frac{5707}{295}$. Adding any edge to $H$ to create $\tilde{H}$, we find that $\kappa(\tilde{H})=\frac{1567}{81}<\kappa(H)$; thus any non-edge for $H$ is a non-Braess edge.

Together these two examples reveal the influence of the number of dominating vertices in determining whether or not a complete multipartite graph is a Braess graph or not.

In the case that $k_{1}=2$, we are able to make a definitive statement.

Theorem 3.4.2. Suppose that $s \geq 2, p \geq 1, G=K_{k_{1}, \ldots, k_{s}} \vee K_{p}$, and that $\min \left\{k_{1}, \ldots, k_{s}\right\}=2$. Then $G$ has a non-Braess edge.

Proof. Set $r=s+p$, and without loss of generality we assume that $k_{1}=2$. Set $\hat{n}=\sum_{j=1}^{s} k_{j}, \hat{\alpha}_{j}=\hat{n}-k_{j}, j=1, \ldots, s$, and $\hat{\gamma}=\sum_{j=1}^{s} k_{j} \hat{\alpha}_{j}$. It now follows via some routine computations that $\alpha_{1}=\hat{\alpha_{1}}+p, \gamma=p^{2}+(2 \hat{n}-1) p+\hat{\gamma}$, and that

$$
\sum_{j=2}^{r} k_{j} \alpha_{j}^{2}=p^{3}+\left(2 \hat{n}+\hat{\alpha}_{1}-2\right) p^{2}+\left((\hat{n}-1)^{2}+2 \hat{\gamma}-2 k_{1} \hat{\alpha}_{1}\right) p+\sum_{j=2}^{r} k_{j} \hat{\alpha}_{j}^{2}
$$


According to Theorem 3.2.5, in order to show that the non-edge corresponding to the independent set of size 2 is a Braess edge, we need to show that

$$
\frac{1}{\gamma n} \sum_{j=2}^{r} k_{j} \alpha_{j}^{2}+\frac{\left(\gamma-2 \alpha_{1}\right)^{2}}{2 \gamma n}-\frac{\gamma}{2 \alpha_{1}}+\frac{\gamma-\alpha_{1}}{\left(\alpha_{1}+2\right) \alpha_{1}}<0 .
$$

Some algebraic manipulations (which use the fact that $\alpha+2=n$ since $k_{1}=2$ ) reveal that (6) is equivalent to

$$
\sum_{j=2}^{r} k_{j} \alpha_{j}^{2}-2 \alpha_{1} \gamma+2 \alpha_{1}^{2}-\gamma<0
$$

Substituting for $\alpha_{j}$ and $\gamma$ in terms of $\hat{\alpha}_{j}, \hat{\gamma}, \hat{n}$ and $p$, and simplifying eventually shows that

$$
\begin{aligned}
& \sum_{j=2}^{r} k_{j} \alpha_{j}^{2}-2 \alpha_{1} \gamma+2 \alpha_{1}^{2}-\gamma= \\
& -p^{3}-\left(2 \hat{n}+\hat{\alpha}_{1}-1\right) p^{2}-\left(3 \hat{n}^{2}-4 \hat{n}\right) p+\sum_{j=2}^{r} k_{j} \hat{\alpha}_{j}^{2}-2 \hat{\alpha}_{1} \hat{\gamma}+2 \hat{\alpha}_{1}^{2}-\hat{\gamma} .
\end{aligned}
$$

Observe that since $\hat{\alpha}_{j} \leq \hat{\alpha}_{1}, j=2, \ldots, s$, we have

$\sum_{j=2}^{r} k_{j} \hat{\alpha}_{j}^{2}-2 \hat{\alpha}_{1} \hat{\gamma}+2 \hat{\alpha}_{1}^{2}-\hat{\gamma}<\sum_{j=2}^{r} k_{j} \hat{\alpha}_{j}^{2}-\sum_{j=2}^{r} k_{j} \hat{\alpha}_{j} \hat{\alpha}_{1}+\hat{\alpha}_{1}\left(-\hat{\gamma}+2 \hat{\alpha}_{1}\right)-\hat{\gamma}<0$.

Inequality (7) follows readily, yielding the desired conclusion.

Next, we show that if there are enough dominating vertices, there is a non-Braess edge for our complete multipartite graph.

Theorem 3.4.3. Suppose that $s \geq 2$ and that $\min \left\{k_{1}, \ldots, k_{s}\right\} \geq 3$. There is a $p_{0} \in \mathbb{N}$ such that if $p \geq p_{0}$, there is a non-Braess edge for the graph $G=K_{k_{1}, \ldots, k_{s}} \vee K_{p}$.

Proof. Fix $p \in \mathbb{N}$, let $G=K_{k_{1}, \ldots, k_{s}} \vee K_{p}$, and form $\tilde{G}$ from $G$ by adding the edge between vertices 1 and 2 . Let $r=s+p$, and set $\hat{n}=\sum_{j=1}^{s} k_{j}$, so that $n=\hat{n}+p$. Let $\hat{\alpha}_{j}=\hat{n}-k_{j}, j=1, \ldots, s$, and $\hat{\gamma}=\sum_{j=1}^{s} k_{j} \hat{\alpha}_{j}$. As in the proof of Theorem 3.4.2, we have $\alpha_{1}=\hat{\alpha_{1}}+p, \gamma=p^{2}+(2 \hat{n}-1) p+\hat{\gamma}$, and

$$
\sum_{j=2}^{r} k_{j} \alpha_{j}^{2}=p^{3}+\left(2 \hat{n}+\hat{\alpha}_{1}-2\right) p^{2}+\left((\hat{n}-1)^{2}+2 \hat{\gamma}-2 k_{1} \hat{\alpha_{1}}\right) p+\sum_{j=2}^{r} k_{j} \hat{\alpha}_{j}^{2} .
$$


From Theorem 3.2.3, the edge between vertices 1 and 2 is a non-Braess edge if and only if

$$
\begin{aligned}
& -\frac{\gamma}{2 \alpha_{1}}+\frac{1}{\gamma}\left[\frac{\left(k_{1}-2\right) \gamma}{2}+\sum_{j=2}^{r} k_{j} \alpha_{j}\left(\frac{\alpha_{j}}{n}+\frac{k_{1}-2}{2}+\frac{(n-2)\left(\gamma-k_{1} \alpha_{1}\right)}{2 n \alpha_{1}}\right)\right]+ \\
& \frac{\gamma-\alpha_{1}}{\alpha_{1}\left(\alpha_{1}+2\right)}<0 .
\end{aligned}
$$

Multiplying (8) by $2 \alpha_{1}\left(\alpha_{1}+2\right) n \gamma$ and the applying some algebraic manipulations, we find that (8) is equivalent to the following:

$$
\begin{aligned}
& -(\gamma+2) \gamma \alpha_{1} n+(n-2)\left(\alpha_{1}+2\right)\left(\gamma-k_{1} \alpha_{1}\right)^{2}+ \\
& \left(k_{1}-2\right) \alpha_{1} n\left(\alpha_{1}+2\right)\left(2 \gamma-k_{1} \alpha_{1}\right)+2\left(\alpha_{1}+2\right) \alpha_{1} \sum_{j=2}^{r} k_{j} \alpha_{j}^{2}<0 .
\end{aligned}
$$

We are going to consider the left side of $(9)$ as a polynomial in $p$, and determine its leading term. Observe that $\alpha_{1}$ and $n$ are linear in $p, \gamma$ is quadratic in $p$, and $\sum_{j=2}^{r} k_{j} \alpha_{j}^{2}$ is cubic in $p$, so that the left side of $(9)$ is of degree at most 6 in $p$. Note further that the leading term of $\left(k_{1}-2\right) \alpha_{1} n\left(\alpha_{1}+2\right)\left(2 \gamma-k_{1} \alpha_{1}\right)$ is $2\left(k_{1}-2\right) p^{5}$, while the leading term of $2\left(\alpha_{1}+2\right) \alpha_{1} \sum_{j=2}^{r} k_{j} \alpha_{j}^{2}$ is $2 p^{5}$.

Next we note that

$$
\begin{aligned}
& -(\gamma+2) \gamma \alpha_{1} n+(n-2)\left(\alpha_{1}+2\right)\left(\gamma-k_{1} \alpha_{1}\right)^{2}= \\
& -2 k_{1} \alpha_{1}^{2} \gamma n+2 n \gamma^{2}-2 \alpha_{1} \gamma^{2}+ \\
& 2 k_{1} \alpha_{1}^{2} \gamma+3 k_{1}^{2} \alpha_{1}^{3} n-\left(4 k_{1}+2\right) \alpha_{1} n \gamma-2 k_{1}^{2} \alpha_{1}^{3}-4 k_{1}^{2} \alpha_{1}^{2} .
\end{aligned}
$$

From 10$)$, we find that the leading term of $-(\gamma+2) \gamma \alpha_{1} n+(n-2)\left(\alpha_{1}+\right.$ $2)\left(\gamma-k_{1} \alpha_{1}\right)^{2}$ is $-2 k_{1} p^{5}$, and hence we deduce that the leading term on the left side of $(9)$ is $2\left(k_{1}-2\right) p^{5}+2 p^{5}-2 k_{1} p^{5}=-2 p^{5}$. We deduce that for all sufficiently large values of $p$, inequality (8) holds, from which the conclusion follows readily.

Next, we consider a special case.

Theorem 3.4.4. Suppose that $s \geq 2, k \geq 3$ and $p \geq 1$, and consider the graph $G=K_{k, \ldots, k} \vee K_{p}$, where the number of independent sets of size $k$ is $s$. 
Then $G$ is a Braess graph if and only if

$$
\begin{aligned}
0 & <-2 p^{5}+[(10-10 s) k-10] p^{4}+\left[\left(-20 s^{2}+40 s-10\right) k^{2}+(6-40 s) k+12\right] p^{3} \\
& +\left[\left(-20 s^{3}+60 s^{2}-36 s+4\right) k^{3}+\left(-60 s^{2}+36 s\right) k^{2}+(24 s-12) k\right] p^{2} \\
& +\left[\left(-10 s^{4}+40 s^{3}-42 s^{2}+12 s\right) k^{4}+\left(-40 s^{3}+54 s^{2}-10 s-4\right) k^{3}\right. \\
& \left.+\left(12 s^{2}-22 s+10\right) k^{2}+(4 s-4) k\right] p \\
& +\left(-2 s^{5}+10 s^{4}-16 s^{3}+10 s^{2}-2 s\right) k^{5}+\left(-10 s^{4}+24 s^{3}-18 s^{2}+4 s\right) k^{4}
\end{aligned}
$$

Proof. Maintaining the notation of Theorem 3.2.3, we find that $r=s+p, n=$ $s k+p, \alpha_{j}=(s-1) k+p, j=1, \ldots, s, \alpha_{j}=n-1, j=s+1, \ldots, r$, and $\gamma=p^{2}+(2 s k-1) p+s(s-1) k^{2}$. An uninteresting computation reveals that $2 \alpha_{1}\left(\alpha_{1}+2\right) n \gamma\left\{-\frac{\gamma}{2 \alpha_{1}}+\frac{1}{\gamma}\left[\frac{\left(k_{1}-2\right) \gamma}{2}+\sum_{j=2}^{r} k_{j} \alpha_{j}\left(\frac{\alpha_{j}}{n}+\frac{k_{1}-2}{2}+\frac{(n-2)\left(\gamma-k_{1} \alpha_{1}\right)}{2 n \alpha_{1}}\right)\right]+\right.$ $\left.\frac{\gamma-\alpha_{1}}{\alpha_{1}\left(\alpha_{1}+2\right)}\right\}$ is equal to the right-hand side of 11 . The conclusion now follows from Theorem 3.2 .3 .

Corollary 3.4.5. Let $k, s, p$ and $G$ be as in Theorem 3.4.4, and suppose that $s \geq 3$. Then each non-edge of $G$ is a non-Braess edge.

Proof. From the hypothesis that $s \geq 3$, the following inequalities are readily established: $-20 s^{2}+40 s-10<0,-20 s^{3}+60 s^{2}-36 s+4<0,-10 s^{4}+40 s^{3}-$ $42 s^{2}+12 s<0,-40 s^{3}+54 s^{2}-10 s-4<0,-2 s^{5}+10 s^{4}-16 s^{3}+10 s^{2}-2 s<0$, and $-10 s^{4}+24 s^{3}-18 s^{2}+4 s<0$. From these inequalities, it follows that on the right-hand side of (11), the coefficients of the $p^{5}, p^{4}, p^{3}, p^{2}$ and $p$ terms, as well as the constant term, are all negative. The conclusion now follows.

Corollary 3.4.6. Suppose that $k \geq 3$ and $p \geq 1$. Then $K_{k, k} \vee K_{p}$ is a Braess graph if and only if

$$
\begin{aligned}
0 & <-2 p^{5}-(10 k+10) p^{4}-\left(10 k^{2}+74 k-12\right) p^{3}+\left(12 k^{3}-168 k^{2}+36 k\right) p^{2} \\
& +\left(16 k^{4}-128 k^{3}+14 k^{2}+4 k\right) p+4 k^{5}-32 k^{4} .
\end{aligned}
$$

Proof. The conclusion follows upon substituting $s=2$ in (11) and simplifying.

Remark 1. Analysing the right-hand side of (12), it is straightforward to determine that if $k>8$, then considered as a polynomial in $p$, the coefficients of the right-hand side of (12) exhibit exactly one sign change. Hence that polynomial in $p$ has at most one positive root, by Descartes' rule of signs. It now follows that if $k>8$ and $K_{k, k} \vee K_{p}$ is a Braess graph, then so is $K_{k, k} \vee K_{q}$ for $q=1, \ldots, p$. 
Remark 2. Suppose that $k_{1}, k_{2} \geq 3$ and that $p \in \mathbb{N}$, and consider the graph $G=K_{k_{1}, k_{2}} \vee K_{p}$. Suppose without loss of generality that $k_{1} \leq k_{2}$. Using techniques similar to those above, it can be shown that adding an edge into the independent set of size $k_{1}$ will increase Kemeny's constant if and only if

$$
\begin{aligned}
& 0<-2 p^{5}+\left[-10 k_{2}-10\right] p^{4}+\left[-20 k_{2}^{2}-40 k_{2}+10 k_{1}^{2}-34 k_{1}+12\right] p^{3} \\
& +\left[-18 k_{2}^{3}\left(-8 k_{1}-50\right) k_{2}^{2}+\left(30 k_{1}^{2}-94 k_{1}+24\right) k_{2}+8 k_{1}^{3}-24 k_{1}^{2}+12 k_{1}\right] p^{2} \\
& +\left[-6 k_{2}^{4}+\left(-16 k_{1}-16\right) k_{2}^{3}+\left(22 k_{1}^{2}-72 k_{1}+4\right) k_{2}^{2}\right. \\
& \left.+\left(16 k_{1}^{3}-40 k_{1}^{2}+10 k_{1}+4\right) k_{2}\right] p \\
& -6 k_{1} k_{2}^{4}+\left(2 k_{1}^{2}-16 k_{1}\right) k_{2}^{3}+\left(8 k_{1}^{3}-16 k_{1}^{2}\right) k_{2}^{2} \equiv f\left(k_{1}, k_{2}\right) .
\end{aligned}
$$

Similarly, adding an edge into the independent set of size $k_{2}$ increases Kemeny's constant if and only if $0<f\left(k_{2}, k_{1}\right)$.

Next, applying some simplifications to $f\left(k_{1}, k_{2}\right)-f\left(k_{2}, k_{1}\right)$, we find that

$$
\begin{aligned}
& f\left(k_{1}, k_{2}\right)-f\left(k_{2}, k_{1}\right)= \\
& \left(k_{1}-k_{2}\right)\left[10 p^{4}+6\left(5 k_{1}+5 k_{2}+1\right) p^{3}\right. \\
& +2\left(13 k_{1}^{2}+32 k_{1} k_{2}+13 k_{1}+13 k_{2}^{2}+13 k_{2}-6\right) p^{2} \\
& +2\left(3 k_{1}^{3}+19 k_{1}^{2} k_{2}+8 k_{1}^{2}+19 k_{1} k_{2}^{2}+24 k_{1} k_{2}\right. \\
& \left.-2 k_{1}+3 k_{2}^{3}+8 k_{2}^{2}-2 k_{2}-2\right) p \\
& \left.+2 k_{1} k_{2}\left(k_{1}+k_{2}\right)\left(3 k_{1}+3 k_{2}+8\right)\right] .
\end{aligned}
$$

Consequently, since $k_{1} \leq k_{2}$, we have $f\left(k_{1}, k_{2}\right) \leq f\left(k_{2}, k_{1}\right)$. We deduce from the above that $G$ is a Braess graph if and only if $(13)$ holds.

We close with a conjecture that is supported in part by Corollary 3.4.5 and Remark 2 .

Conjecture 3.4.7. Suppose that $r \geq 2, p \in \mathbb{N}$, and $\min \left\{k_{1}, \ldots, k_{r}\right\} \geq 3$. Let $G=K_{k_{1}, \ldots, k_{r}} \vee K_{p}$. Then $G$ is a Braess graph if and only if:

i) $r=2$; and assuming (without loss of generality) that $k_{1} \leq k_{2}$,

ii) (13) holds.

\section{Acknowledgements}

Yuxiang Hu's research was supported by a University of Manitoba Faculty of Science Undergraduate Research Award. Steve Kirkland's research is supported in part by NSERC Discovery Grant RGPIN-2019-05408. The authors are grateful to the referee, whose comments helped to improve the presentation. 


\section{References}

[1] D. Braess, Über ein Paradoxon aus der Verkehrsplanung, Unternehmensforschung 12 (1969), 258-268.

[2] D. Braess, A. Nagurney and T. Wakolbinger, On a paradox of traffic planning, Transportation Science 39 (2005), 446-450.

[3] A. Brouwer and W. Haemers, Spectra of Graphs, Springer-Verlag, New York, 2012.

[4] E. Crisostomi, S. Kirkland and R. Shorten, A Google-like model of road network dynamics and its application to regulation and control, International Journal of Control 84 (2011), 633-651.

[5] A. Ghayoori and A. Leon-Garcia, Robust network design, 2013 IEEE International Conference on Communication (ICC), Budapest, 2013, 2409-2414.

[6] A. Jadbabaie and A. Olshevsky, On performance of consensus protocols subject to noise: Role of hitting times and network structure, 2016 IEEE 55th Conference on Decision and Control (CDC), Las Vegas, 2016, 179 184.

[7] J. Kemeny and J. Snell, Finite Markov Chains, Springer-Verlag, New York, 1976.

[8] S. Kirkland and M. Neumann, Group Inverses of M-matrices and Their Applications, CRC Press, Boca Raton, 2013.

[9] S. Kirkland and Z. Zeng, Kemeny's constant and an analogue of Braess' paradox for trees, Electronic Journal of Linear Algebra 31 (2016), 444464.

[10] V. Moosavi and G. Isacchini, A Markovian model of evolving world input-output network, PLoS ONE 12 (2017): e0186746. 